website : http:// jsci.utq.edu.iq

Email: utjsci@utq.edu.iq

\title{
Effect of Quantum Dots Density and temperature on Quantum Dot Laser Characteristics
}

\author{
Ra'ed M. Hassan* Chassib A. Emshary Hassan A. Sultan \\ Dept. of Physics- College of Education for Pure Science- University of Basrah \\ *Email: raed_m_hassan@yahoo.com
}

\begin{abstract}
$\underline{\text { Abstract }}$
Detailed theoretical study of the effects of surface density of quantum dots on the dynamics of quantum dot lasers (QDLs) is given. Temperature dependence of the threshold current density, confined level occupancy, internal loss coefficient, optical confinement layer (OCL), characteristic temperature and lowest excitation energy with the variation of internal loss constant component, root mean square of relative QD-size fluctuations, mean size of QDs and optical confinement factor are studied.
\end{abstract}

Keywords: Quantum dot lasers, Surface density of QDs, GaInAsP/InP laser, Characteristic temperature.

\section{تأثير كثافة النقاط الكمية و الحرارة على خصائص ليزر النقطة الكمية}

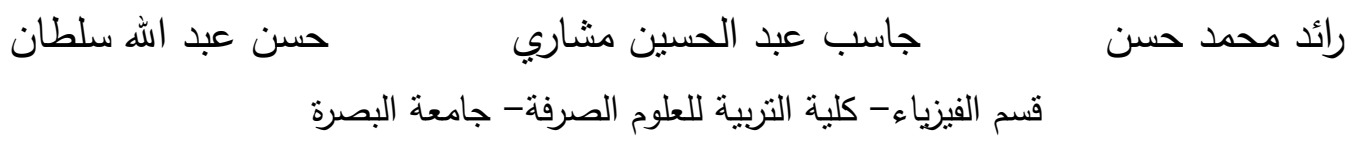

الخلاصة

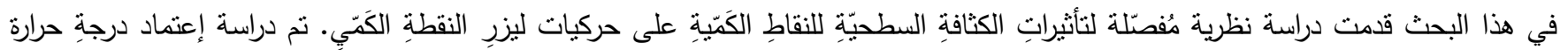

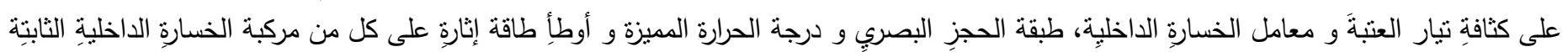

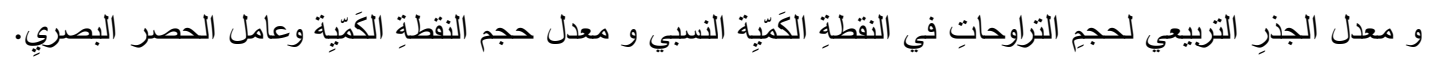

\section{Introduction}

Quantum dot (QD) semiconductor lasers are of particular interest due to certain advantages over the conventional semiconductor quantum well lasers viz. narrow gain spectra, significant low threshold currents and weak temperature dependence. As a consequence of quantum confinement in all the three dimensions the energy spectra of electrons and holes are discrete in QDs. Transitions between electron and hole levels are analogous to those between the exactly discrete levels of individual atoms. According to this reason, the behavior of structures with QDs have generated much interest as a new class of artificially structured materials with tunable energies through varying the compositions and sizes of discrete atomic-like states that are ideal for use in laser
structures.Internal optical losses are present in all types of semiconductor lasers. Such losses affect the laser operating characteristics, increasing their threshold current density and decrease its differential efficiency $[1,2]$. As a result of lower values of optical confinement factor in these lasers the effect of internal loss in stronger in comparison with bulk semiconductor lasers [1] . Several mechanisms can contribute to the internal loss viz. $1^{\text {st; }}$; free carrier absorption in the optical confinement layer (OCL) and in the cladding layers, $2^{\text {nd }}$; inter-valence band absorption, $3^{\text {rd }}$; carrier absorption in the quantum confined active region itself, and $4^{\text {th }}$; scattering at rough surfaces and imperfections of the waveguide[1]. The key characteristics of a semiconductor laser are the light-current. The current 
density at which lasing starts is defined as the threshold current density $j_{t h}$. The temperature dependence of $j_{t h}$ can be described by the following exponential function, $j_{t h} \alpha \exp \left(\frac{T}{T_{0}}\right)$ [3], where $T_{0}$ is called the characteristic temperature. The higher is the characteristic temperature, $T_{0}$, the higher is the temperature stability of $j_{t h}$ [3]. Lowering $j_{t h}$ and imposing its temperature stability have been the important objectives in the development of semiconductor lasers [4].The present work gives the theoretical results of various parameter effects on the dynamics of QD laser viz. the threshold current densities, $j_{t h}$, in QD $\left(j_{Q D o t}\right)$ and OCL $\left(j_{O C L}\right)$, internal loss coefficient, confined electron level occupancies in QD, QD and OCL characteristic temperatures, OCL free-electron density and QD lowest excitation energy.

\section{The Theoretical Model}

Several mechanisms can contribute to the internal loss such as the free-carrier absorption in the OCL and scattering at rough surfaces and imperfections in the waveguide [5].All these mechanisms can be conveniently grouped into two categories one dependent on the carrier density in the OCL and the other is independent. The expression for the saturation value of the modal gain in QDL, $G_{m}$ is [6,7]

$$
G_{m}=\frac{\sigma \hbar \Gamma \xi_{\text {ODot }}}{4 M_{\text {ODDot }} \tau_{D} \rho\left(\delta^{\varepsilon} E^{\varepsilon}+\delta^{h} E^{h}\right)}\left(\frac{\lambda}{k}\right)^{2}
$$

where $\sigma$ is the QD-size distribution function( $\sigma=1 / \sqrt{2 \pi}$ for the Gaussian distribution), $\hbar$ is Planck constant, $\Gamma$ is the optical confinement factor in the QD layer, $\xi_{Q D o t}$ is the surface density of QDs, $\delta^{e, h}=-\frac{\partial E^{e, h}}{\partial\left(\ln M_{Q D o t}\right)}$, which is assumed to be the same for all modes, $M_{Q D o t}$ is the mean size of QDs, $\tau_{D}$ spontaneous radiative recombination time, $\rho$ is the root mean square (RMS) of relative QD-size fluctuations, $\quad E^{e, h}$ are the electron/hole quantized energy levels in a mean-sized QD, $\lambda$ is the wavelength of the main mode in vacuum, and $k$ is the group index of the dispersive OCL material. $T_{0}^{Q D o t}$ and $T_{0}^{O C L}$ are two components of the characteristic temperature $T_{0}$ which is defined similarly to $T_{0}$ for $j_{Q D o t}$ and $j_{O C L}$ respectively [6]:

$\frac{1}{T_{0}}=\left(\frac{j_{Q D o t}}{T_{0}^{\text {ODot }}}+\frac{j_{O C L}}{T_{0}^{O C L}}\right) / j_{t h}$

$j_{Q D o t}$ and $j_{O C L}$ are the components of $j_{t h}$ which are associated with the recombination in QDs and in OCL respectively, where

$j_{t h}=j_{\text {QDot }}+j_{\text {OCL }}$.

these components are given as $[6,8]$ :

$$
\begin{aligned}
& j_{Q D O t}=\frac{e \xi_{Q D O t}}{\tau_{Q D o t}} f_{Q D O t}^{s} f_{Q D O t}^{h} \\
& j_{O C L}=e \wp_{O C L} R_{O C L} \bar{f}_{O C L}^{b} \bar{f}_{O C L}^{h} \cdots
\end{aligned}
$$

where $\boldsymbol{e}$ is the electronic charge, $\tau_{Q D o t}$ is the spontaneous radiative recombination time in QDs, $f_{Q D o t}^{e, h}$ are the confined-electron/hole level occupancies in QDs at the lasing threshold respectively, $\bar{f}_{O C L}^{e, h}$ are the freeelectron / hole densities in the OCL at the lasing threshold respectively, $\wp_{O C L}$ is the OCL thickness and $R_{O C L}$ is the radiative constant for the OCL material.

The equality of the gain to the loss (lasing threshold condition) can be written as [9]:

$$
G_{m}=\frac{\frac{1}{L} \ln \left[\frac{1}{r}\right]+k_{\text {loss }}}{2 f_{\text {DDot }}^{\varepsilon}-1}
$$

where $r$ is the mirror facet reflectivity and $L$ is the cavity length. $k_{\text {loss }}$ can be written as the sum of two components which are the overall internal loss coefficients, one constant $\left(k_{0}\right)$ and the other increasing with the OCL electron density $\left(\bar{f}_{O C L}^{e}\right)[5,10]$;

$k_{\text {ioss }}=k_{0}+\Theta_{\text {eCs }} \bar{f}_{\text {OCL }}^{\varepsilon}$.

where $\Theta_{\text {ecs }}$ is the effective cross section for the internal absorption loss processes. When $\Theta_{e c s}=0$, i.e. the absence of the electron-density-dependent internal loss, so the level occupancy is immediately obtained from Eq. (5) to be independent of temperature [3]:

$$
f_{Q D \omega_{0}}^{\varepsilon}=\frac{1}{2}\left[1+\frac{\frac{1}{L} \ln \left[\frac{1}{r}\right]+k_{0}}{G_{m}}\right] \ldots
$$

where $f_{Q D o t}^{e}$ are the confined-electron level occupancies in QDs at the lasing threshold $\left(f_{Q D o t}^{e}=f_{Q D o t_{0}}^{e}\right.$ at 
$\left.\Theta_{e c s}=0\right)$. As seen from Eq. (4), the electron-densitydependent internal loss couples $\bar{f}_{O C L}^{e}$ and $f_{Q D o t}^{e}$ make $f_{Q D o t}^{e}$ and $j_{Q D o t}$ also temperature dependent in view of the temperature dependence of $\bar{f}_{O C L}^{e}$. Thus, $T_{0}^{Q D o t}$ becomes finite. The expression for $f_{Q D o t}^{e}$ is [5];

$f_{\text {QDot }}^{\varepsilon}=\frac{1}{2}\left(1+f_{\text {QDoto }}^{\varepsilon}-W\right)-\sqrt{\frac{1}{4}\left(1+f_{\text {QDots }}^{\varepsilon}-W\right)^{2}-f_{\text {QDot }}^{\varepsilon}}$

Where

$$
W=\bar{f}_{O C L_{0}}^{\varepsilon} \frac{\Theta_{e c s}}{2 G_{m}}
$$

In Eq. (8), due to the carrier-density-dependent internal loss formally plays a role similar to that of the violation of charge neutrality in QDs that affect the characteristic temperature, $T_{0}$, via its effect on the level occupancy $f_{\text {QDot }}^{e}[8,9]$.

The characteristic temperature component $T_{0}^{Q D o t}$ can be expressed as $[10,11]$;

$$
\frac{1}{T_{0}^{O D D t t}}=\frac{\frac{W}{T}\left(3+\frac{2 \varepsilon^{\varepsilon}}{T}\right)}{\sqrt{\left(1+f_{\text {ODot }}^{b}-W\right)^{2}-4 f_{\text {ODDot }}^{e}}} .
$$

the free-carrier density is strongly affective; therefore, the carrier-density-dependent internal loss also alters the temperature dependence of $j_{O C L}$ at the lasing threshold. The position effect of a characteristic temperature from the critical point is important to determine the QD laser operation. In this case, the expression for $T_{0}^{O C L}$ is [11];

$\frac{1}{T_{0}^{O C L}}=\frac{1}{2}\left[\frac{3}{T}+\frac{4 \varepsilon^{\varepsilon}}{T^{2}}+\frac{2}{T_{0}^{O D D t}\left(1-f_{\text {QDot }}^{\varepsilon}\right.}\right]$.

The $T$-dependence of the free-carrier densities $\left(\bar{f}_{O C L}^{e, h}\right)$ acts as the major source of such dependence of $j_{t h}$ for which the carrier distribution below, and at the lasing threshold is described by the equilibrium statistics (relatively high $T) \quad[5,11,12] . \quad \bar{f}_{O C L}^{e, h}$ depends exponentially on $T$ [5];

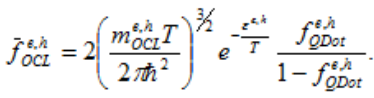

$T$ is the temperature measured in units of energy where respectively, $m_{O C L}^{e, h}$ are the electron / hole effective masses in the OCL and $\varepsilon^{e, h}$ are the electron/hole excitation energies from a QD to the OCL. The carrier density $\bar{f}_{O C L}^{e, h}$ in the OCL with smaller $E^{e}$, that are easier for the carriers to escape. Higher $f_{Q D o t}^{e}$ and $k_{\text {loss }}$ are also strongly controlled by the excitation energy $E^{e}$ from OCL to the QD [12]. There is the Lowest Excitation Energy $E_{\text {low }}^{e}$ below which no lasing is attainable in the structure. An explicit expression is apparent for $E_{\text {low }}^{e}[11]$;

$$
E_{i o w}^{e}=T \ln \left[\frac{2 \Theta_{e c s}\left(\frac{m_{o c L}^{\varepsilon}}{2 \pi \hbar^{2}}\right)^{3 / 2}}{\left(\sqrt{2 G_{m}}-\sqrt{G_{m}+k_{i o s s}+\frac{1}{L}} \ln \left[\frac{1}{r}\right]\right)^{2}}\right]
$$

\section{Results and Discussion}

In what follows, a GaInAsP / InP hetero-structure lasing near $1.55 \mu \mathrm{m}$ will be considered. The parameters used in the calculations are as follows: surface density of the QDs, $\xi_{\text {QDot }}=5 \times 10^{10} \mathrm{~cm}^{-2}$, the cavity length, $L=$ $1.628 \mathrm{~mm}$, the mirror facet reflectivity, $r$, equals 0.32 , the mean size of the QDs, $M_{Q D o t}=150 \mathrm{~A}^{\circ}$, electron quantized energy levels in a mean-size (measured from the corresponding band edge), $E^{e}=6 . \mathrm{meV}$, RMS of the relative QD-size fluctuations, $\rho=0.05$, the overall internal loss coefficient, $k_{\text {loss }}=3.0 \mathrm{~cm}^{-1}$, the effective cross section for the internal absorption loss processes, $\Theta_{e c s}=2.67 \times 10^{-17} \mathrm{~cm}^{2}$ and the optical confinement factor in the QD layer, $\Gamma=0.06$. The constant part of the overall internal loss coefficient $k_{0}$ will take the values $(0,1,3,6$ and 10). Other parameters mentioned above will take the following values: $\rho=0.04-0.07, M_{Q D o t}$ $=120-200\left(\mathrm{~A}^{\circ}\right), \Gamma=0.04-0.08$ and $\xi_{Q D o t}=4-8\left(\times 10^{10}\right.$ $\mathrm{cm}^{-2}$ ). In Fig. 1, the threshold current density, $j_{t h}$ and one of its components i.e. $j_{O C L}$ in the OCL are drawn against temperature for a number of the surface density of QD values. The usual trend of the relation is clear, i.e. as the surface density is increased both $j_{t h}$ and $j_{O C L}$ increase monotonically reaching maximum values at higher temperatures. The maximum operating temperature increases with an increasing surface density too. The line connecting the bold dots represents the maximum operating temperature in the QD microcavity. Fig. 2 shows the internal loss coefficient, $k_{\text {loss }}$, against temperature for a number of the surface density given in the previous figure. It is clear that as the surface density increases so does the loss coefficient marking 
the high operating temperature as shown by the straight solid line. By the same trend, as the surface density of QDs increases, the confined electron level occupancy, decreases too with increasing temperature, see Fig. 3. The straight line in this figure marks the trend of the reduction of confined-electron level occupancy with temperature.

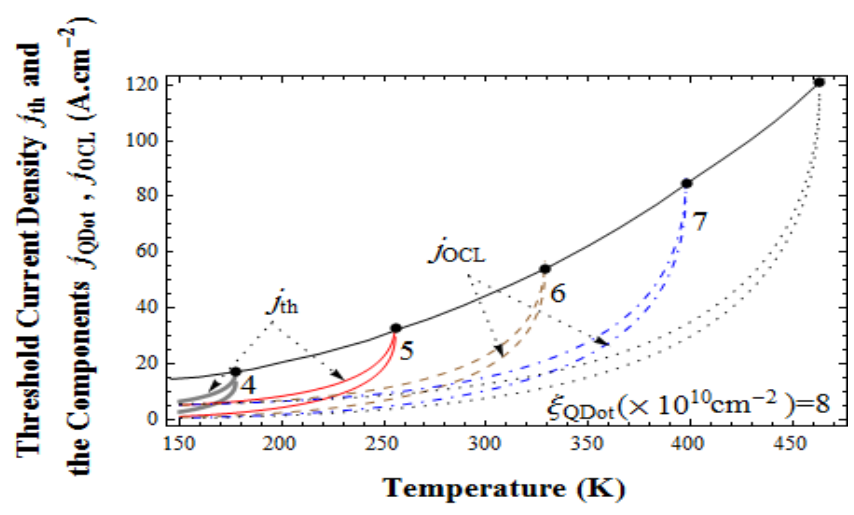

Fig. 1. Threshold current density, $j_{t h}$, and OCL component, $j_{O C L}$ vs. temperature, $T$, for different values of surface density of QDs; $\xi_{Q D o t}\left(\times 10^{10} \mathrm{~cm}^{-2}\right)=$ 4, 5, 6, 7 and 8. The top curve marks the QD microcavity maximum operating temperatures. The parameters used for GaInAsP/InP QDL [4]: $L=1.628$ $\mathrm{mm}, r=0.32, \lambda=1.55 \mu \mathrm{m}, M_{Q D o t}=150 \mathrm{~A}^{\mathrm{o}}, E^{e}=6$. $\mathrm{meV}, \quad \rho=0.05, \quad k_{\text {loss }}=3 . \mathrm{cm}^{-1}$ and $\Theta_{\text {ecs }}$ $=2.67 \times 10^{-17} \mathrm{~cm}^{2}, \Gamma=0.06$.

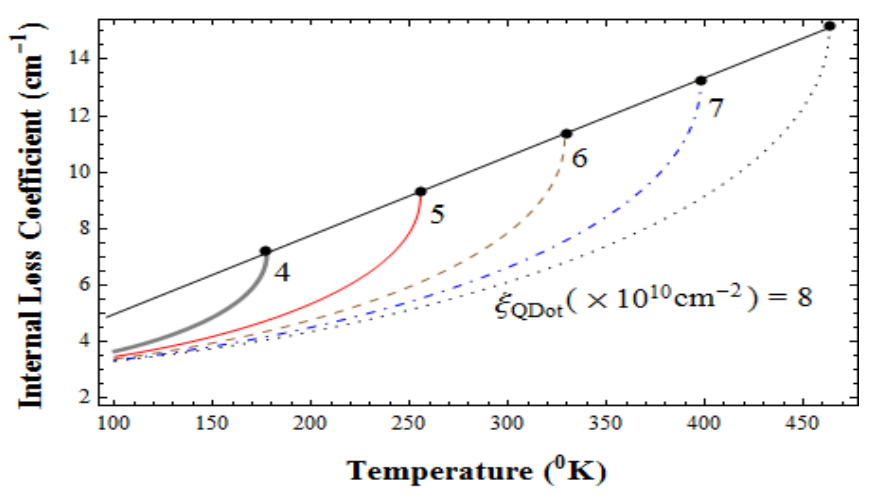

Fig. 2. Internal loss coefficient, $k_{\text {loss }}$ vs. temperature, $T$, for different values of surface density of QDs; $\xi_{Q D o t}$ $\left(\times 10^{10} \mathrm{~cm}^{-2}\right)=4,5,6,7$ and 8 . The upper straight line marks the QD micro-cavity maximum operating temperature. The same parameters values are used in Fig. 1.

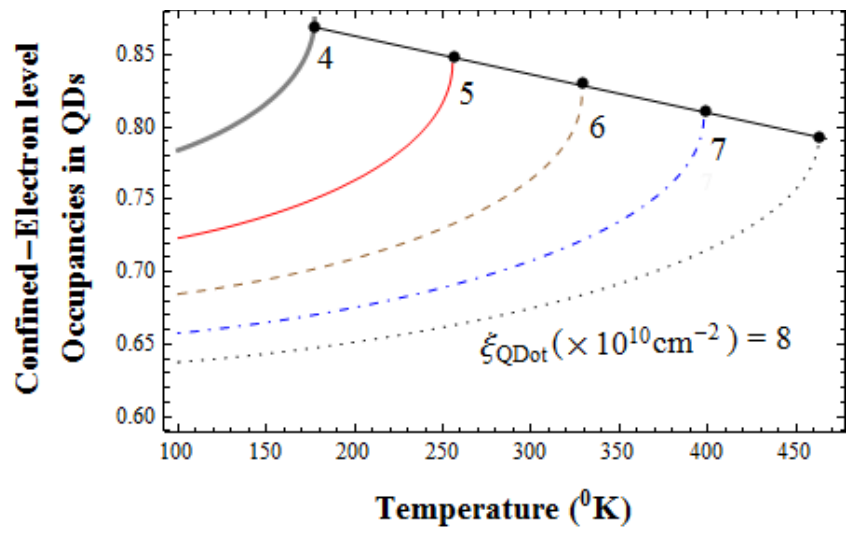

Fig. 3. Confined-carrier level occupancy in QDs, $f_{Q D o t}^{e}$ vs. temperature, $T$, for different values of surface density of QDs; $\xi_{Q D o t}\left(\times 10^{10} \mathrm{~cm}^{-2}\right)=4,5,6,7$ and 8 . The upper straight line marks the QD micro-cavity maximum operating temperature. The same parameter values are used as in Fig. 1.

By drawing a line perpendicular to the horizontal axis, one can figure out the amount of reduction of a level occupancy with an increasing surface density. Fig. 4 gives the relation between QD and OCL characteristic temperatures and temperature for the same amount of surface density values chosen in the previous figures. As the surface density increases the characteristic temperature $T_{0}^{Q D o t}$ shows a clear decrease towards zero while $T_{0}^{O C L}$ decreases towards zero after a small increase in each of the chosen surface density values. By drawing a line perpendicular to the horizontal axis, shows a high increase of the characteristic temperature in QD with an increasing surface density. Fig. 5 shows the relation between QD laser characteristic temperature against the surface density of QDs for different values of the constant part of the loss coefficient $\left({ }^{k_{0}}\right)$, Fig. 5(a), for different values of RMS of relative QD-size fluctuations ( $\rho)$, Fig. 5(b), for different values of mean size of QDs ( $M_{Q D o t}$ ), (see Fig.5(c)) and last for different values of optical confinement factor $(\Gamma)$, Fig. 5 (d). As expected with the reduction of losses $T_{0}^{\text {QDot }}$ has increased (Fig. 5 (a)), it increased with the reduction of QD size fluctuation (Fig. 5 (b)), it increased as expected, with a decreasing mean size fluctuation (Fig. 5 (c)) and it increases with an increasing optical confinement factor (Fig. 5 (d)). 


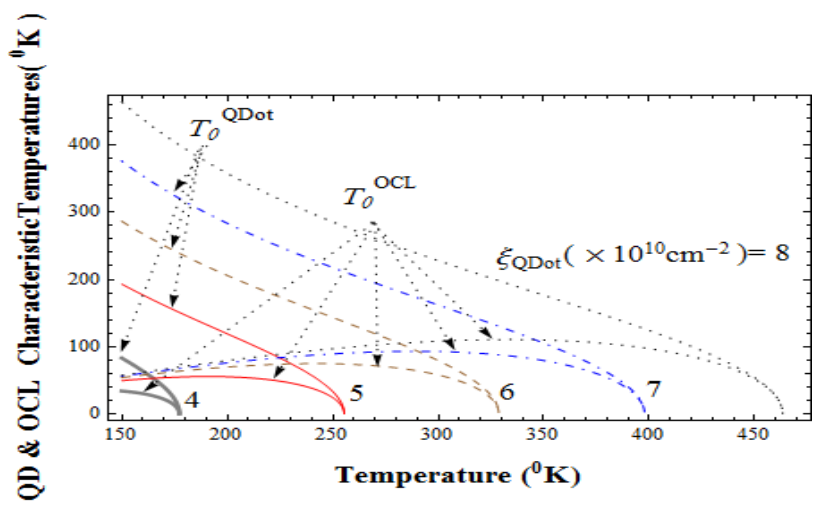

Fig. 4. QD and OCL characteristic temperature vs. temperature; $T$ for different values of surface density of QDs; $\xi_{Q D o t}\left(\times 10^{10} \mathrm{~cm}^{-2}\right)=4,5,6,7$ and 8 , using the same parameters mentioned in the previous figures.

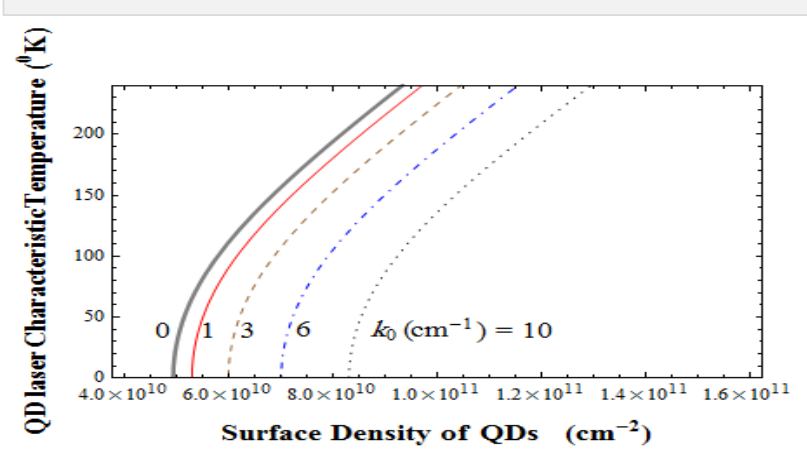

(a)

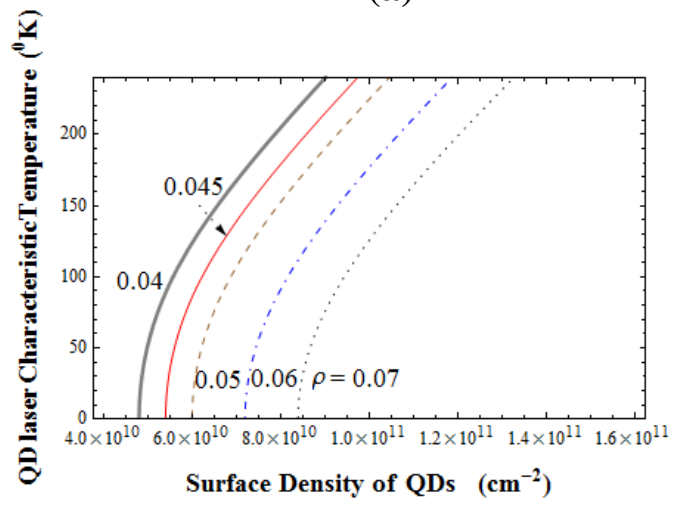

(b)

Fig. 5. Laser Characteristic Temperature, $T_{0}$ vs. Surface Density of QDs processes for; (a) Constant component of internal loss; $k_{0}\left(\mathrm{~cm}^{-1}\right)=0,1,3,6$ and 10 respectively, (b) RMS of relative QD-size fluctuations; $\rho=0.04,0.045,0.05,0.06$ and 0.07 respectively, (c) Mean size of QDs; $M_{Q D o t}\left(\mathrm{~A}^{\circ}\right)=120,135,150,180$ and 200 respectively, and (d) Optical confinement factor in the QD layer; $\Gamma=0.04,0.05,0.06,0.07$ and 0.08 .

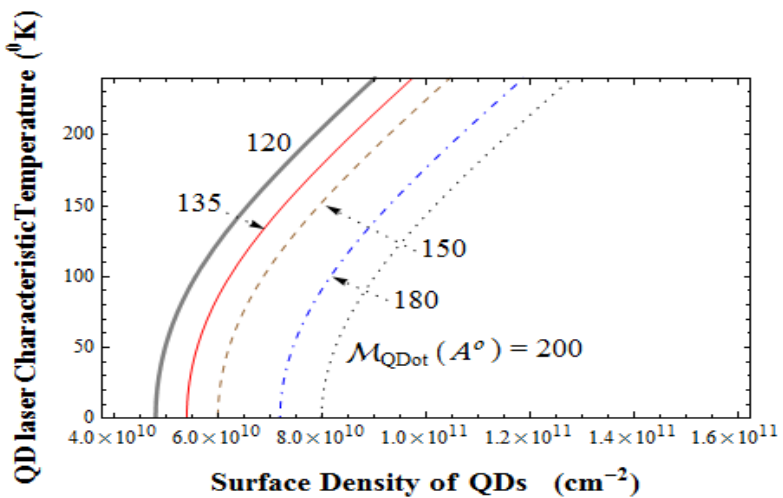

Fig. 5. (c)

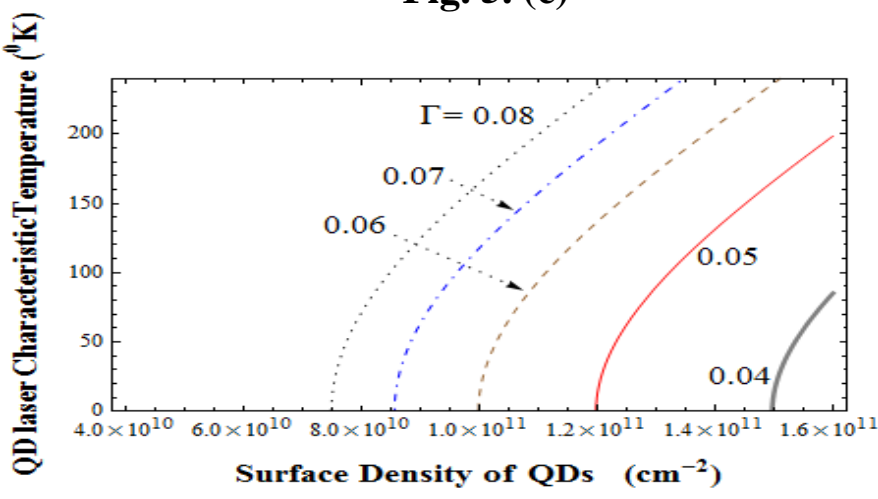

Fig. 5. (d)

In Fig. 6 the relation between OCL free-electron density and temperature is given where as expected the former increased with an increasing surface density of QDs as the straight solid line indicates. At last, the relation between the QD lowest excitation energy and surface density of QDs for a variation of the constant loss coefficient, $k_{0}$, (Fig. 7 ((a)), RMS of the relative QD-size fluctuations, $\rho$, (Fig. 7 (b)), the mean size of QDs $M_{\text {QDot }}$ (Fig.7(c)) and a variation of the optical confinement factor, $\Gamma$, (Fig. 7 (d)). The increase of surface density of QDs decreases the QD lowest excitation energy for each $k_{0}$ chosen. Drawing a line perpendicular to the horizontal axis reveals the fact that for a fixed surface density of QDs the QD lowest excitation energy increases with the increase of $k_{0}$. The same thing can be concluded for $\rho$ and $M_{Q D o t}$ while by increasing the confinement factor $(\Gamma)$ the QD lowest excitation energy decreases. 


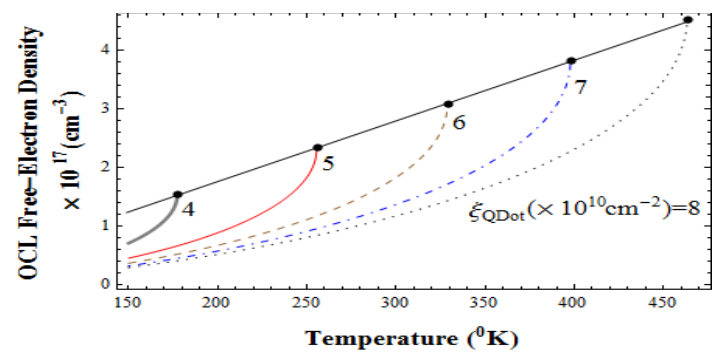

Fig. 6. OCL free-electron density, $\bar{f}_{O C L}^{e}$ vs. temperature, $T$ for different values of surface density of QDs; $\xi_{\text {QDot }}\left(\times 10^{10} \mathrm{~cm}^{-2}\right)=4,5,6,7$ and 8 . The straight line marks the QD micro-cavity maximum operating temperature.

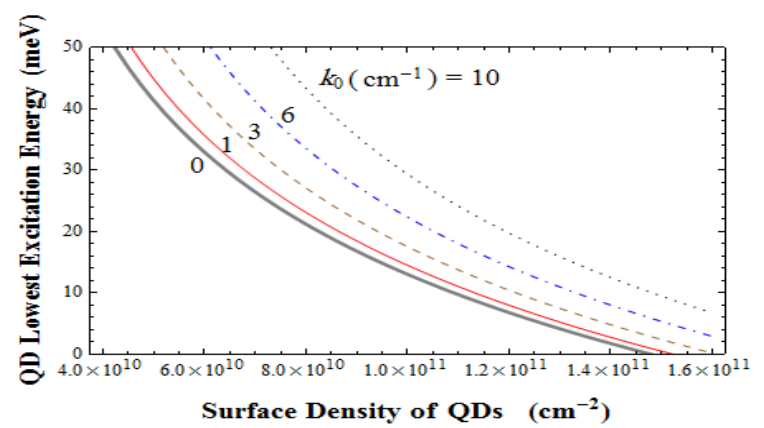

(a)

Fig. 7. QD lowest excitation energy, $E_{\text {low }}^{e}$ vs. Surface Density of QDs for different values of; (a) Constant component of internal loss; $k_{0}\left(\mathrm{~cm}^{-1}\right)=0,1,3,6$ and 10 respectively, (b) RMS of relative QD-size fluctuations; $\rho=0.04,0.045,0.05,0.06$ and 0.07 respectively, (c) Mean size of QDs; $M_{Q D o t}\left(\mathrm{~A}^{\mathrm{o}}\right)=120,135,150,180$ and 200 respectively, and (d) Optical confinement factor in the QD layer; $\Gamma=0.04,0.05,0.06,0.07$ and 0.08 .

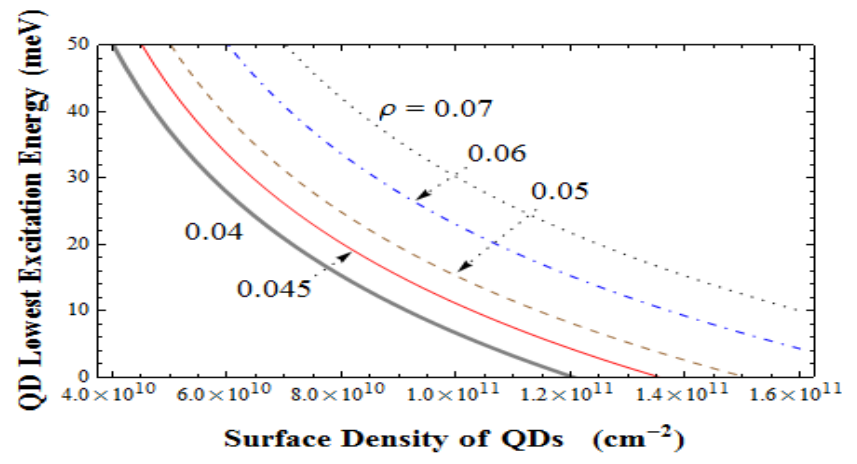

Fig. 7. (b)

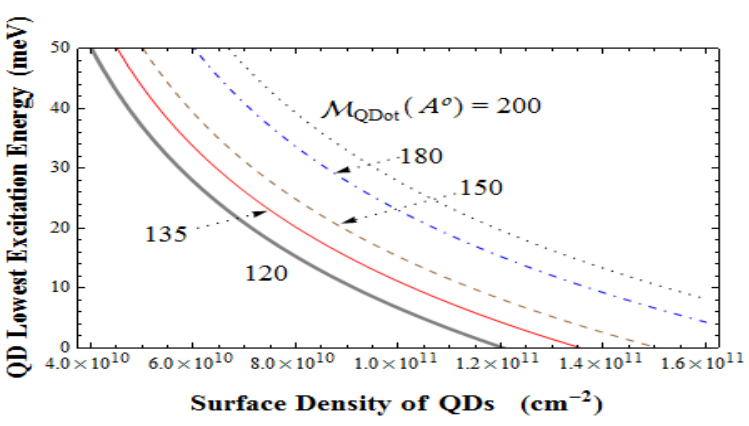

Fig. 7. (c)

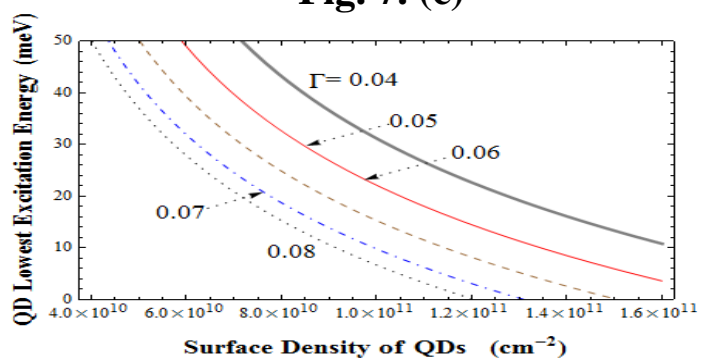

From Fig. 1 and by imagining lines perpendicular to the temperature axis from each bold point, we can see that the operating temperature depends directly on the surface density in each case. For $\xi_{Q D o t} \geq 6 \times 10^{10} \mathrm{~cm}^{-2}$, it becomes quite easy to run the QDL above room temperature, although the internal loss coefficient increases with increasing $\xi_{Q D o t}$ and the confined electron level occupancy decreases (Figs. 2 and 3 respectively). With increasing temperature (Fig. 4), the effect of the carrier density-dependent internal loss becomes more strongly manifested hence, $T_{0}^{Q D o t}$ curve decreases for each surface density chosen. $T_{0}^{Q D o t}$ suffers the same decrease although it is milder. By fixing the surface density in each part of the figure of Fig. 5, we can see the following: $T_{0}$ increases with decreasing $k_{0}, \rho$, and $M_{Q D o t}$ but it increases with an increasing $\Gamma$.In an ideal QDL, the threshold current, $j_{t h}$ , should remain unchanged with the temperature and the characteristic temperature $T_{0}$ should be infinitely high. This would be the case if the overall injection went into QDs, and the recombination current in QDs would be temperature-independent. In actual QDLs, the carriers are first injected from the cladding layers into the optical confinement layer OCL (which includes the wetting layer), then captured into the QDs. The presence of carriers in the OCL results in the 
recombination therein. Hence the recombination processes both in QDs and in the OCL control $j_{t h}$ and its T-dependence. From the equations given in the introduction, we can write $T=\left[\frac{\partial \ln j_{t h}}{\partial t}\right]^{-1}$.

The less temperature-sensitive is the $j_{t h}$, the higher is $T_{0}$ [3].

\section{Conclusion}

The present study shows that various parameters of the quantum dot lasers play vital role in affecting the output of QDLs, these parameters are internal loss coefficient, confined-electron level occupancy, etc. We conclude the followings: the maximum threshold current density increases exponentially with the increase of surface density of quantum dots. Maximum internal loss increases linearly with the surface density of QDs, confinedelectron level occupancy shows reversal dependence on surface density of QDs while the temperature range of lasing operation increases with the increase of surface density of QDs. To achieve the same QDL characteristics with increase of internal loss, we need to increase the surface density of QDs. Once more to achieve the same temperature the RMS of the relative need to be increased, and the same thing is needed when varying the mean size of QDs, while we need to decrease the optical confinement factor. As quantum dot density increase the maximum optical confinement layer linearly increases as the temperature increases. Increasing the internal losses increases the values of QD lowest excitation energy for the same surface density of QDs values, and to achieve the same behavior as the value of RMS of relative QD-size fluctuations increases we need to increase the values of mean size of QD while the optical confinement layer need to be decreased to achieve the same values of QD lowest excitation energy.

\section{References}

[1] G. P. Agrawal and N. K. Dutta. "Long-wavelength semiconductor lasers". CVan Nostrand, NewYork (1986).

[2] P. S. Zory. "Quantum well lasers". (BBoston, MA. Ed., Academic (1993).

[3] L. Jiang. PhD thesis, Virginia Polytechnic Institute and State University, USA,(2008).

[4] R. M. Hassan, PhD thesis; "Theoretical Study of Turn-on Dynamics and Lasing Operation Characteristics of Semi-conductor Quantum Dot Lasers". College of Education, University of Basrah, Basrah, Iraq (2010).

[5] L.V. Asryan and S. Luryi, IEEE Journal of Quantum Electronics, 40 (2004) 833-843.

[6] L. V. Asryan and R. A. Suris, Semicond. Sci. Technol., 11 (1996) 554-567.

[7] L. V. Asryan, M. Grundmann, Nikolai N. Ledentsov, Oliver Stier, Robert A. Suris, and Dieter Bimberg. IEEE Journal of Quantum Electronics, 37 (2001) 418-425.

[8] L. V. Asryan and R. A. Suris, IEEE Journal of Quantum Electronics, 34 (1998) 841-850.

[9] L. V. Asryan and R. A. Suris, IEEE Journal of Selected Topics in Quantum Electronics, 3 (1997) 148-157.

[10] R. M. Hassan, C. A. Emshary, S. I. Easa, Journal of Thi-Qar Science, 3

(2011) 146-156.

[11] L. Jiang and L.V. Asryan. Laser Physics Letters, 4 (2007) 265-269.

[12] D. S. Han and L. V. Asryan. Solid-State Electronics, 52 (2008) 1674-1679. 